\title{
Kernos
}

Revue internationale et pluridisciplinaire de religion grecque antique

1 | 1988

Varia

\section{Homerische Begräbnissbraüche}

\section{Georg Pedaros}

Édition électronique

DOI : $10.4000 /$ kernos. 112

ISSN : 2034-7871

\section{Éditeur}

Centre international d'étude de la religion grecque antique

\section{Édition imprimée}

Date de publication : 1 janvier 1988

ISSN : 0776-3824

\section{Référence électronique}

Georg Pedaros, « Homerische Begräbnissbraüche », Kernos [En ligne], 1 | 1988, mis en ligne le 31 janvier 2011, consulté le 01 mai 2019. URL : http://journals.openedition.org/kernos/112 ; DOI : $10.4000 /$ kernos. 112 
Kernos, 1(1988), p. 195-206.

\section{HOMERISCHE BEGRÄBNISBRÄUCHE}

Die Begräbnisbräuche der Menschen aller Zeiten und Kulturen spiegeln ihre Lebenseinstellung wieder und bringen ihre Auffassung vom Leben sowie vom Leben nach dem Tode zum Ausdruck.

Während in vielen östlichen Religionen das Leben als Erleben der Todesbewußtheit erfahren wird, war bei den homerischen Menschen die Liebe zum irdischen Leben so tief verwurzelt, daß selbst der mystische Glaube der archaischen Epoche an das Weiterleben der Seele nach dem Tod die Zeit nicht überdauern konnte und schon im 5. Jahrhundert zu verfallen begann. Der Anschauungsweise des homerischen Menschen entspricht das Bewußtsein vom zentralen Wert des Lebens und das Verlangen nach dem Genuß dieses Lebens sowohl im Sinnlichen als auch im Bereich des Idealen. Das Leben und die Existenz des homerischen Menschen auf Erden ist ein großes und nicht ersetzbares Gut, zumal es Voraussetzung für alles andere ist ${ }^{1}$. Nur der Ehre könnte vielleicht ein dem Leben gleich hoher Wert zugekommen sein. Damals liebten die Menschen das Leben auf einfache und selbstverständliche Weise, ohne jeden metaphysischen Vorbehalt. Das Leben betrachteten sie als Licht und den Tod als Dunkelheit. Als sich die Achäer wegen des Rauches, den Zeus um den leblosen Körper des Patroklos aufsteigen ließ, in Gefahr sahen, verlangte Aias von Zeus nicht, sie vor dem Tode zu bewahren; er forderte lediglich, daß er den Nebel aufhebe und sie im Licht vernichte :

Z $\varepsilon \hat{v} \pi \dot{\alpha} \tau \varepsilon \rho$,

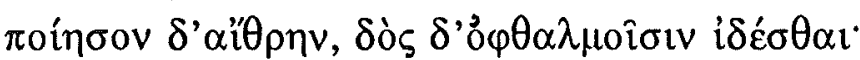

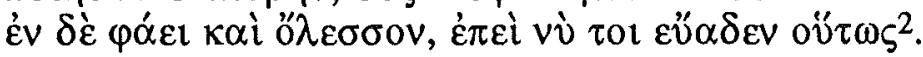

Die homerische Vorstellung vom Wert des Lebens ist absolut. Deshalb wird sie ergänzt durch das Gegenbild zum Leben, der

1 Cf. E. ROHDE, Psyche : Seelenkult und Unsterblichkeitsglaube der Griechen, 2. Aufl., Leipzig-Tübingen, 1898 (Darmstadt, 1974), I, S. 2.

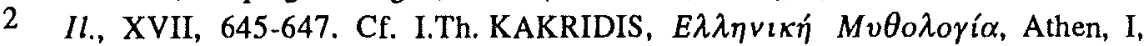
S. 34,36 . 
grenzenlosen Ablehnung des Todes als der Negation des Lebens. Noch war kein Weg entdeckt, das Leben und seine Negation entweder durch die Religion oder durch die Philosophie zu versöhnen. Für alle Menschen der Welt gibt es keine größere Agonie als die Erwartung des unvermeidbaren Todes; für den homerischen Menschen jedoch war darüber hinaus der Gedanke, mit dem Tod versinke er in die Nicht-Existenz, besonders schmerzlich. Der $\theta \alpha ́ v \alpha \tau o \zeta$ ist die eigentliche $\mu$ oîp $\alpha$ des Menschen, die schwere Last in seinem Leben ${ }^{3}$. Gewiß berichten die Epen von Menschen, die den Tod überwandten, sowie von Menschen, die unsterblich wurden (z.B. verspricht Kalypso dem Odysseus die Unsterblichkeit), aber die Erwartung des Todes übt eine so starke Wirkung aus, daß nicht einmal die Götter sich ihr entziehen können (Fall des Sarpedon). Die aristokratische Gesellschaft Homers schrieb ihren Göttern die Unsterblichkeit zu, die sie selbst so sehr ersehnte. Sie markiert die Grenze zwischen der Vollkommenheit der Götter und der Unvollkommenheit der Menschen. Wenn gelegentlich die Götter den Menschen scheinbar einige Möglichkeiten zur Erlangung der Unsterblichkeit gewähren, so bereuen sie dies letzlich immer (Il., XVII, 443 ff.).

Für das homerische Denken ist das Leben nach dem Tode so öde und leer, daß es kaum von der Nicht-Existenz entfernt ist. Die

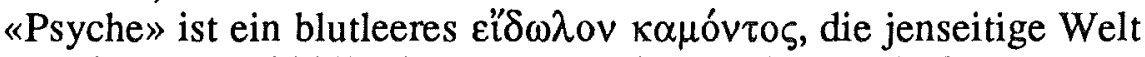
nur ein mattes Abbild des gegenwärtigen Lebens. Die Seele trennt sich nach dem Tod vom Körper und steigt rasch in den Hades hinab, tritt aber erst dort ein, nachdem der Körper verbrannt ist und nicht vorher. Im Hades gibt es keinerlei Denken, kein Fühlen und keine Kraft. Nie wieder kann die Seele in die Welt der Lebenden zurïckkehren, und daher wird ihr auch keine Verehrung erwiesen und kein Kult eingerichtet. Diese Auffassung sowie die Tatsache, daß der Leichnam in der mykenischen Epoche nicht verbrannt wurde, führt zu' dem Schluß, daß die homerischen Epen in schärfstem Gegensatz zu den Bestattungsbräuchen der mykenischen Welt stehen, die sie

3 Der homerische Mensch hat eine genaue Vorstellung von dieser Gesetzmäßigkeit sowie von der Ordnung, die ihr zu Grunde liegt und die er $\alpha \hat{l} \sigma \alpha$, $\mu \circ \hat{\rho} \rho \alpha$ nennt. Kń $\rho$, die Göttin des Todes, gehört in einen anderen Zusammenhang. 
angeblich beschreiben ${ }^{4}$. Betrachten wir aber zunächst die homerischen Bestattungsbräuche analytisch ${ }^{5}$.

Die erste rituelle Handlung nach dem Tod einer geliebten Person ist die Waschung des Leichnams und dessen Einsalben mit Öl : $\kappa \alpha \grave{ }$

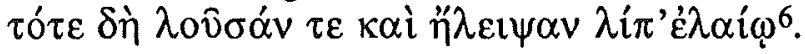

Die Gefährten des Achilles vollzogen diese Handlung selbst am toten Patroklos, wogegen Achilles seine Sklavinnen anwies, den toten Hektor $\mathrm{zu}$ waschen und mit Ölen zu salben : $\delta \mu \omega \grave{\alpha} \varsigma$

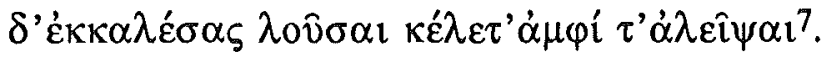

Der Gebrauch von Wasser ist uns dadurch bekannt, daß Odysseus die $\chi 0{ }^{\prime} \varsigma^{8}$ vergießt, um die Seelen anzulocken. In den Elysischen Feldern ist Wasser im Überfluß vorhanden, Tantalos dagegen wird im Hades von Durst gepeinigt. Als beständiges Element wird Wasser auch im babylonischen Totenkult verwendet. Nach dem Gilgamesch-Epos haben die Könige im Hades das Privileg, kaltes Wasser trinken zu dürfen. Der Wasserkrug fehlte niemals auf den älteren babylonischen Gräbern, wobei sich daneben ein Kelch aus Ton oder Bronze befand, den der Tote benutzen sollte. Der Durst des Toten ist auch in Syrien und Palästina bekannt, wo der Vollzug des Trankopfers mit Wasser auf Grabmälern dargestellt ist. Die Gräber der Bibel und der Ras-Shamra waren mit einem System von Kanälen versehen, über das den Toten Wasser und andere

4 Für die gegensätzlichen Auffassungen siehe Anm. 18; Cf. G.E. MYLONAS,

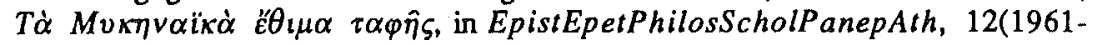
1962), S. 291 ff.

5 Den Grabes- und Totenkult der mykenischen Zeit untersuchte hauptsächlich E. ROHDE in seinem noch keineswegs uberholten Werk Psyche (cf. supra Anm. 1); er wies auf die Spuren eines in der Zeit Homers bereits vergangenen Stadiums der Totenkult-vorstellungen hin. Die jüngere Bibliographie fuhren wir im Verlauf des Textes auf.

$6 \quad$ Il., XVIII, 350.

$7 \quad$ Il., XXIV, 582.

$8 \quad O d ., \mathrm{XI}, 24 \mathrm{ff}$. 
Trankopfer zugeführt wurden. In Ägypten bot man dem Toten in der Zeit des Alten Reiches nicht nur Wasser zum Waschen, sondern auch zum Trinken dar; bei den Kulthandlungen der Osiris-Mysterien in der Zeit des Mittleren Reiches gewann die Verwendung von Wasser sogar eine außerordentliche Bedeutung; es wurde zum Medium der geistigen Auferstehung. Eine entsprechende Bedeutung findet sich im Alten Testament, wenn die lebensspendende Feuchtigkeit in Bezug zur Auferstehung der Toten gesetzt wird ${ }^{9}$. Derselbe Brauch ist folglich in verschiedenen Kulturen verschiedener Epochen anzutreffen, mag auch die jeweilige Bedeutung Unterschiede aufweisen.

Das Öl diente außer bei der Salbung auch als Grabbeigabe. Bei der Verbrennung der Leiche des Patroklos stellte Achilles zwei tönerne Gefäße auf, wovon das eine mit Honig und das andere mit Öl gefüllt ist ${ }^{10}$. Wie Plutarch überliefert ${ }^{11}$, wird Öl auch noch in späterer Zeit bei den Trankopfern im Totenkult verwendet. Bekanntlich ist es noch heute bei Begräbnissen im Gebrauch; es bildet also ein Grundelement der Totenfeier, zumindest in der griechischen Provinz.

Das Schließen von Augen und Mund erfolgte vermutlich aus praktisch-ästhetischen Gründen; es sollte nicht unter die Bestattungsbräuche gefaßt werden.

Darauf folgt die Prothesis des Toten und der yóos, der Klagegesang. Die relativ lange andauernde Aufbahrung der Helden Hektor, Patroklos und Achilles - bekanntlich blieb Hektors Leichnam neun Tage unbestattet, der des Achilles sogar 17 - führte zu der Auffassung, daß sich aus den Epen möglicherweise eine Art Präparierung der Leichen nachweisen lasse. Diese Ansicht versuchte man damit zu stützen, daß die Freunde des Patroklos diesen

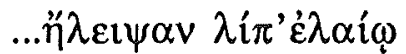

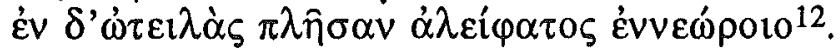

9 A.T., $I s ., 26,19$.

10 Il., XXIII, 170.

11 PLUT., Vit. Arist., 23, 1.

12 Il., XVIII, 350-351. 
Eine Mumifizierung nach ägyptischem Vorbild jedoch gibt es in den Homerischen Epen nicht. Das Verb $\tau \alpha \rho \chi v ́ \varepsilon ı v$, das ursprünglich die Bedeutung von «bewahren, erhalten, konservieren» hat, wird bei Homer mit der Bedeutung «bestatten» verwendet, wie sich an der. Episode des Sarpedon nachweisen 1 läßt ${ }^{13}$. Die ägyptische Technik der Mumifizierung und die mykenischen Goldsmasken haben die Funktion, die Auslöschung der körperlichen Identität zu verhindern, während die Brandbestattung gerade diese erstrebt. Denn in den obengenannten Kulturen hat die Verbrennung der Toten nicht mehr den Sinn, den sie vermutlich zur Zeit des Nomadentums hatte, nämlich jene Voraussetzungen zu schaffen, die es dem Toten erlauben würden, mittels ritueller Behandlung seiner Knochen wiedergeboren zu werden. Darüber hinaus bedeutet die Konservierung des toten Körpers in der mykenischen Zeit auch die Erhaltung seiner Kräfte, seiner Macht - gefährliche Dinge für die Nachlebenden ${ }^{14}$.

Ein ständig wiederkehrender Begriff des Totengesangs in den Epen ist róos:

$$
\begin{aligned}
& \alpha \hat{v} \tau \grave{\alpha} \rho \quad ' A \chi \alpha \imath \imath \grave{l}
\end{aligned}
$$

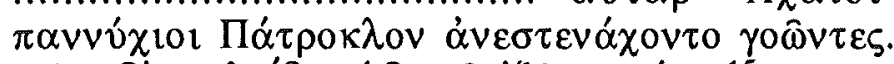

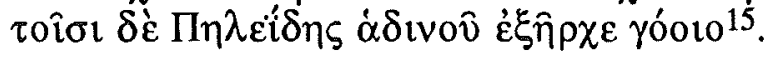

Ursprünglich artikuliert sich die Klage im rhythmischen Wort, um dann zum Versmaß und zur Melodie fortzuschreiten. Auf diese Weise entsteht die weitverbreitete Form der metrischen

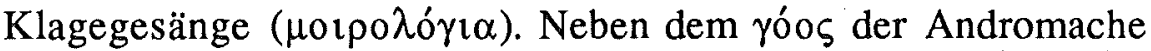

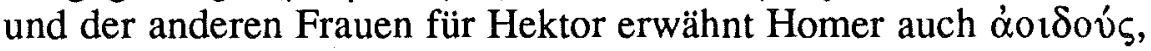
die eine metrische Klage darstellen :

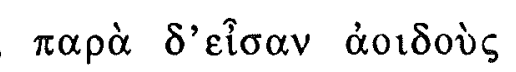

$\theta \rho \eta ́ v \omega v \dot{\varepsilon} \xi \dot{\alpha} \rho \chi 0 v \varsigma$,

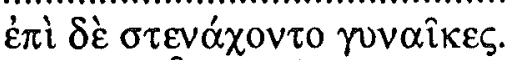

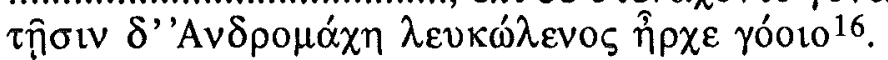

13 Il., XVI, 456.

14 A. SCHNAUFER, Frühgriechischer Totenglaube, in Spudasmata, 20(1970), S. 8 ff.

15 Il., XVIII, 314-316.

16 Il., XXIV, $720 \mathrm{ff}$. 


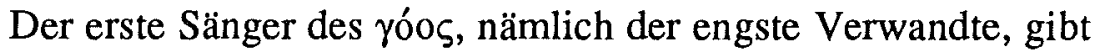
seinen Part an andere Verwandte weiter. Der formale Charakter des Klagegesangs kommt auch dadurch zum Ausdruck, daß man gewöhnlich die gefangenen. Feinde zur Klage verpflichtete. So müssen z.B. trojanische Sklavinnen, von Achilles gezwungen, am Leichnam des Patroklos klagen ${ }^{17}$.

Thematischer Inhalt des Klagegesangs ist keinesfalls der Verlust der Existenz - sie wird im Gegenteil weiterhin vorausgesetzt -, sondern eher die Entleerung der Existenz.

Der bedeutendste und zentrale Akt im Zusammenhang mit der Bestattung des Toten ist zweifellos die Leichenverbrennung. "Alle anderen Bräuche, die vor oder nach der Bestattung gepflegt werden, haben nicht den fundamentalen Stellenwert des Aktes, durch den der Leichnam vernichtet wird ${ }^{18}$.

In den Homerischen Epen ist die Verbrennung die einzig erwähnte Form der Bestattung. Eine Ausnahme bildet vielleicht der Fall des Sarpedon ${ }^{19}$, welcher - was nicht vergessen werden darf als Sohn des Zeus eine unterschiedliche Behandlung erfahren mußte. Im übrigen bedeutet, wie wir gesehen haben, das Verb $\tau \alpha \rho \chi v ́ \omega$ in dem Vers

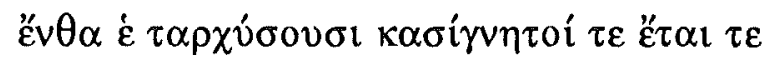

$\tau \hat{v} \mu \beta \omega \tau \varepsilon \sigma \tau \dot{\eta} \lambda \eta ̣ \tau \varepsilon \cdot \ldots . . .{ }^{20}$

mit Sicherheit «bestatten». Folglich kommt es möglicherweise auch im Falle von Sarpedon nach seiner Überführung in die Heimat, nach Lykien, zur Brandbestattung.

In den Epen werden also die Toten verbrannt. Die mykenischen Fürsten werden dagegen beerdigt, während in der darauffolgenden

17 Il., XVIII, 339 ff.

18 M. ANDRONIKOS, 'O 17(1962), S. 42.

19 Il., XVI, $666 \mathrm{ff}$.

20 Il., XVI, 674-675, 456-457. 
(nach-homerischen) Zeit beide Bestattungsformen nebeneinander bestehen. Hier stoßen wir auf einen der vieldiskutierten Widersprüche der homerischen Archäologie.

Für die Brandbestattung bei Homer liefert uns der Fall des Patroklos ${ }^{21}$ eine vollständige Beschreibung; diesem entnehmen wir überdies die meisten Informationen zu den homerischen Bestattungsbräuchen. Eine kurze Darstellung ist daher lohnenswert :

Die Leiche des Patroklos wird mit dem Haar seiner Gefährten bedeckt, wobei sein brüderlicher Freund Achilles den ganzen Vorgang beaufsichtigt und auch selbst sein blondes Haar niederlegt

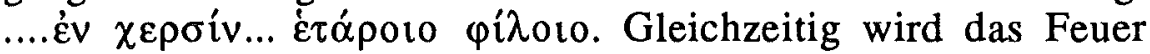
angerichtet. Es folgt die Aufbahrung des Leichnams auf dem Holzsto $\beta$ und die Schlachtung der Tiere, die mit dem Toten ver-

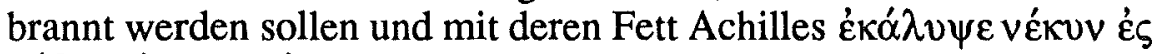
$\pi \delta ́ \delta \alpha \varsigma \dot{\varepsilon} \kappa \kappa \varepsilon \varphi \alpha \lambda \hat{\eta} \varsigma$.

Zusammen mit dem Leichnam werden auch zwei mit Öl uns Honig gefüllte Amphoren verbrannt, dazu vier Pferde, zwei Hunde

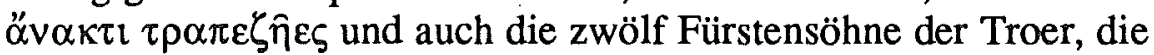
Patroklos mit eigener Hand im Kampf getötet hatte. Der erste Teil des Rituals, in dessen Verlauf Anrufungen und Trankopfer vollzogen werden, endet mit dem Entzünden des Feuers.

Nachdem der Leichnam verbrannt ist, wird das Feuer mit Wein gelöscht; die Knochen werden in einer goldenen Phiale gesammelt und der Feuerplatz verwandelt sich in eine Grabstätte, auf der eine Stele als $\gamma \dot{\varepsilon} \rho \alpha \zeta \theta \alpha v o ́ v \tau \omega v$ errichtet wird. Damit ist der rituelle Akt der Bestattung abgeschlossen.

Das Totenmahl ist ebenfalls ein Teil der Bestattungsfeier, aber es ist nicht bekannt, ob es vor oder nach der Bestattung eingenommen wurde. Das Totenmahl zu Ehren des Patroklos fand vor der Bestattung sıatt, das Mahl zu Ehren des Hektor dagegen im Anschluß an die Bestattung. Es scheint aber unabdingbar gewesen zu sein, denn sogar Orest gab zu Ehren seiner Mutter und des Aegisth nach deren Tod ein feierliches Mahl.

21 Il., XXII, 128-177. 
Leichenspiele schließlich, die letzte Kulthandlung im Bestattungsritus, kommen in den Epen nur bei der Bestattung des Patroklos und des Achilles vor; auch Nestor erwähnt sie als eine Erinnerung aus seiner Jugendzeit bei der Bestattung des Amaryngeus.

Über die Bedeutung der verschiedenen Lebewesen und Gegenstände, die zusammen mit dem Leichnam verbrannt wurden, aber auch über den Sinn der Verbrennung selbst, besteht in der Forschung keine Einigkeit. Die Verbrennung der Tiere wurde von Rohde folgendermaßen gedeutet :

Der ganzen Erzăhlung [der Ilias -d.Verf.] liegt die Vorstellung zu Grunde, $\mathrm{da} B$ durch Ausgiessung fliessenden Blutes, durch Weinspenden und Verbrennung menschlicher und thierischer Leichen die Psyche eines jüngst Verstorbenen erquickt, ihr Groll besănftigt werden könne ${ }^{22}$.

Eine gegensätzliche Auffassung, wonach «Zweck der Schlachtung von Tieren [...] die Gewinnung von Fett war, das zur Verbrennung beihelfen sollte» 23 , kann nicht begründen, warum diese Tiere so angeordnet wurden, daß sie mit dem Toten zusammen verbrannten, wie dies bei Pferden und Hunden der Fall war. Die richtige Deutung ist vielmehr, daß es sich um Darbietungen zur Erfreuung und Erbauung ihres einstigen Herrn handelte. Die Niedermetzelung und Verbrennung der zwölf trojanischen Geiseln ist meines Erachtens kein Aufleben der Sitte des Menschenopfers, wie Rohde und andere zunächst behaupteten. In einem fortgeschrittenen Stadium der Zivilisation konnte unmöglich der uralte barbarische Brauch des Menschenopfers zur Geltung gelangen. Im übrigen liefert uns Homer selbst die zutreffende Deutung, indem er

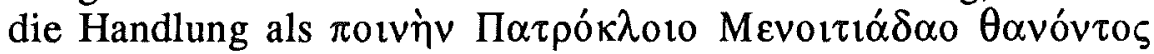
charakterisiert $^{24}$.

22 ROHDE, Psyche, I, S. 17.

23 G.E. MYLONAS, 'O PhilosScholPanepAth, 4(1953-1954), S. 267.

24 Il., XXI, 28. Cf. MYLONAS, op. cit., S. 268. 
Die meisten der obengenannten Bestattungsbräuche sind gleichermaßen bei den Mykenern wie auch in den Epen zu finden. Unbestreitbar unterscheidet jedoch der Brauch der Verbrennung des Leichnams die Welt Homers von Mykene in Bezug auf die Auffassungen vom Leben nach dem Tode. Die bisher bekannten Beigabenfunde an den archäologischen Stätten führen uns zu dem Schluß, daß die Beziehung zwischen dem Toten und den Lebenden von der Idee bestimmt war, der Tote bestehe als «lebender Leichnam» weiter ${ }^{25}$. Dies bedeutet, daß der Tote im Bewußtsein der Nachlebenden nicht einfach nur weiterexistiert, sondern auch über Kräfte und Macht verfügt und damit die Erfüllung seiner Forderungen durchsetzen kann. Diese Überzeugung macht den Brauch der Grabbeigaben verständlich; ihr Zweck war, die Bedürfnisse des Toten im jenseitigen Leben zu befriedigen. Die Angst vor dem Fluch und der Rache des Toten bei seiner eventuellen Rückkehr zwang die Mykener, die Forderung des Toten nach einer Totengabe gewissenhaft $\mathrm{zu}$ erfüllen ${ }^{26}$. Diese Beziehung zwischen den Nachlebenden und dem Toten ist jedoch nicht von langer Dauer und scheint abzubrechen, sobald sich der Leichnam völlig aufgelöst hat ${ }^{27}$. Der «lebende Leichnam» existiert nicht mehr; er ist in einen Haufen von Knochen zerfallen und hat seine Kraft und Macht eingebüßt, welche ihm vorher noch die Einforderung der Grabbeigaben ermöglicht hatten. Er flößt keinen Respekt mehr ein und kann gewiß keine Forderungen mehr stellen.

Der Fall der zwei Säuglingsleichen aus Grab III in Mykene wird als Versuch gedeutet, ihr Weiterleben im Diesseits mittels der goldenen Masken und umhüllenden Goldbleche zu ermöglichen, da sie viel $\mathrm{zu}$ früh gestorben waren ${ }^{28}$. Ein Weiterleben war generell das erstrebte Ziel, wenn die Gräber Goldbeigaben enthielten (Grab IV); dies betraf aber hauptsächlich die Mitglieder fürstlicher Geschlechter und steht nicht im Widerspruch zum Glauben der Mykener an ein Leben nach dem Tod. Auch der Altar, den Schliemann über Grab IV

25 M.P. NILSSON, Geschichte der griechischen Religion, München, 1955 2 , S. 41 .

26 Cf. SCHNAUFER, op. cit., S. $8 \mathrm{ff}$.

27 G.E. MYLONAS, Burial Customs, in A.J.B. WACE - F.H. STUBBINGS ed., A Companion to Homer, London, 1962, S. 484. Cf. SCHNAUFER, op. cit., S. 11 .

28 G. MYLONAS, Ancient Mycenae, Princeton, New Jersey, 1957, S. 139. 
entdeckt hat - wenn dort überhaupt seine ursprüngliche Lage war -, ist kein Beweis für einen fortdauernden Totenkult in mykenischer Zeit. Im übrigen ist der Altar das einzige sichere Zeugnis für diese Auffassung ${ }^{29}$. Neu in der Zeit der Tholos-Gräber ist, daß der Tote einen Wohnraum verlangt. Die Art der Prothesis bleibt dieselbe.

Die Vorstellungen des homerischen Menschen vom Leben nach dem Tod wurden bereits oben kurz ausgeführt. Auf die Leichenverbrennung, die den wichtigsten Unterschied zwischen mykenischen und homerischen Bestattungsbräuchen darstellt, möchten wir genauer eingehen. Es stellen sich zwei grundlegende Fragen : Woher kam diese Bestattungsform ? Welche Ursachen haben sie hervorgerufen?

Es wurde die Auffassung vertreten, daß der Brauch der Leichenverbrennung von indogermanischen Nomadenstämmen nach Griechenland mitgebracht wurde. Die archäologischen Befunde liefern jedoch keinen Beweis für diese These ${ }^{30}$. Nach der Einwanderung der griechischen Stämme ist vielmehr die Beerdigung der Toten die einzige durchgeführte Bestattungsform. Es bleibt demzufolge unsicher, ob die homerischen Epen an frühere Bestattungsriten der mykenischen Zeit erinnern oder ob die Brandbestattung, die uns in den Epen begegnet, zeitlich verschoben die Praxis der geometrischen Epoche wiedergibt.

Eine andere Auffassung besagt, da $\beta$ «von den Troern auch die Achäer während ihres Aufenthalts in der Troas den Brauch [der Leichenverbrennung, d.Verf.] übernommen und adaptiert haben. [...] Nach ihrer Rückkehr nach Griechenland gaben die Achäer den fremdartigen Brauch auf und führten wieder die Erdbestattung durch $^{31}$ ».

29 G. MYLONAS, The Cult of the Dead in Helladic Times, in Studies presented to D.M. Robinson, I, Saint Louis, 1951, S.97. Cf. SCHNAUFER, op. cit., S. 14, Anm. 44.

30 M. ANDRONIKOS, Totenkult, in Archaeologia Homerica, III, 4, Göttingen, 1968 , S. 52 ff.

31 G.E. MYLONAS, in EpistEpetPhilosScholPanepAth, 4(1953-1954) (cf. supra Anm. 23), S. 283. 
Diese Interpretation kann jedoch kaum als befriedigend gelten, denn «sie betrachtet die homerischen Epen wie historische Texte ${ }^{32}$ ». Meines Erachtens gibt es aber einen noch gewichtigeren Einwand gegen diese Deutung. Unter religionswissenschaftlichem Aspekt läßt sich nur mit Schwierigkeit die Annahme akzeptieren, ein Volk oder der Teil eines Volkes würde einen Brauch nur aus Gründen der Zweckmäßigkeit übernehmen, ohne daß dieser Brauch zuvor tief in die Glaubenswelt dieses Volkes eingedrungen ist; ebensolche Schwierigkeiten bèreitet die Annahme, daß ein Brauch zu einem bestimmten zeitlichen Moment aufgegeben wurde. Die Übernahme des Brauchs der Leichenverbrennung, der nur von den ionischen Griechen stammen könnte, ist nun tatsächlich ein Vorgang, der an wesentliche Glaubensinhalte rührt.

Es wurde bereits festgestellt, daß bei den Mykenern die Bedürfnisse des Toten bis zum völligen Zerfallen der Leiche befriedigt werden mußten. Der homerische Mensch dagegen, in dessen Vorstellungswelt der Tod jeglicher metaphysischer Gewißheit entbehrt, übernimmt den Brauch der Leichenverbrennung als ein rettendes Mittel zur sicheren Entfernung des Toten aus der Welt der Lebenden. Mit derselben psychologischen Begründung versuchte auch schon Rohde, das Aufkommen der Brandbestattung zu erklären ${ }^{33}$.

Über die Ausbreitung des Brauchs der Leichenverbrennung in Ionien lassen sich aufgrund des geringen Befundes keine sicheren Aussagen treffen. Wir wissen aber heute, daß die Leichenverbrennung von Anatolien aus in den griechischen Raum eingedrungen ist und daß bereits die Hethiter zwischen 1800 und 1600 v.Chr. diesen Brauch pflegten ${ }^{34}$. Wahrscheinlich verbreitete sich die Brandbestattung zunächst an den Küsten Kleinasiens und Syriens und erreichte dann weiter westlich gelegene Gebiete.

Wir sind der Überzeugung - trotz aller eventuell gegensätzlicher Meinungen -, daß die homerischen Bestattungsbräuche aus der geometrischen Zeit stammen, als das griechische Denken begann,

32 M. ANDRONIKOS, in Hellenica, 17(1962) (cf. supra Anm. 18), S. 44 ff.

33 ROHDE, Psyche, I, S. 30.

34 SCHNAUFER, op. cit., S. 49. 
sich aus den Fesseln einer magischen Deutung der Welt zu befreien und kraft des Logos den Weg für Philosophie, Geschichte und Wissenschaft im allgemeinen bereitete. Sicher begegnen wir vielen der homerischen Bräuche auch schon in mykenischer Zeit, aber diese Bräuche finden sich in allen Perioden der Geschichte der griechischen Welt; viele davon überlebten im Volksglauben sogar bis heute, nicht nur in Griechenland.

E1. Venizelou 218,

Georg PEDAROS

Elioupolis,

GR - 16341 ATHEN 